I NVESTI GACI ÓN

\title{
Eventos adversos observados años después del tratamiento con factor de transferencia
}

\author{
Adverse events occurred some years after the application \\ of a transfer factor treatment
}

MSc. María Aida Cruz Barrios,' MSc. J uan Antonio Furones Mourelle," Lic. Yainet Medina Magaña, "I Dra. I vette de la Fuente Sanz, ${ }^{\text {IV }}$ Lic. Lídice Pérez Nicola, ${ }^{v}$ Lic. Yalexis Díaz Viamontes ${ }^{\mathrm{vI}}$

'Facultad de Medicina "Miguel Enríquez". La Habana, Cuba.

"Escuela Nacional de Salud Pública, La Habana, Cuba.

III Facultad de Enfermería "Lidia Doce". La Habana, Cuba.

IV Dirección Municipal de Salud, municipio "10 de Octubre". La Habana, Cuba.

$\checkmark$ Dirección Provincial de Salud. La Habana, Cuba.

VI Hospital "Freyre de Andrade". La Habana, Cuba.

\section{RESUMEN}

Introducción: el factor de transferencia es un inmunoestimulante que se emplea en varias enfermedades, su seguridad en las condiciones de la práctica médica no se ha establecido.

Objetivos: identificar eventos adversos presentados años después de finalizado el tratamiento con factor de transferencia y determinar la relación entre su utilización y los eventos observados.

Métodos: estudio observacional, retrospectivo, de fármacovigilancia activa. El universo estuvo constituido por 413 pacientes tratados con este fármaco entre los años 2001 y 2004 en 11 hospitales de La Habana. El periodo de observación abarcó los 6 a 8 años posteriores al tratamiento. Los pacientes fueron entrevistados acerca de la presencia de infecciones, cáncer y otros eventos, en su hogar. Si un evento era la muerte, se entrevistaba al familiar para registrar causa y fecha.

Resultados: se entrevistaron 356 pacientes, 66 (18,53\%) presentaron al menos un evento adverso, $21(31,8 \%)$ en mayores de 60 años. Se registraron 87 eventos adversos, $8(9,2 \%)$ fueron infecciones como hepatitis $B$, hepatitis $C$, foliculosis, mononucleosis y verrugas vulgares, $1(1,1 \%)$ cáncer y $78(89,7 \%)$ otros eventos. Su relación con la administración del fármaco resultó condicional en 64 (73,6 \%) pacientes e improbable en $21(24,1 \%)$, en esta última categoría se incluyeron las 
infecciones y la mayoría de los eventos mortales.

Conclusiones: los eventos adversos que se registraron en los pacientes, años después de haber recibido tratamiento con factor de transferencia, no se consideran relacionados con su administración, aunque necesitan ser vigilados pues la información obtenida en este estudio debe ser contrastada con un mayor número de observaciones y estudios observacionales controlados.

Palabras clave: factor de transferencia, reacciones adversas, eventos adversos, farmacovigilancia, farmacoepidemiología.

\section{ABSTRACT}

Introduction: the transfer factor is an immunostimulant that is used to treat several diseases but its safety in the medical practice has not been assured yet.

Objectives: to identify the adverse events occurred some years after the end of a transfer factor treatment and to determine the association of the use and the observed events.

Methods: observational active drug surveillance study. The universe of study was 413 patients who were treated with the transfer factor from 2001 to 2004 in eleven hospitals located in La Habana province. The observation period ranged 6 to 8 years after the treatment. The patients were interviewed at home on the occurrence of infections, cancer or any other events. If some death occurred, then a family member was interviewed to record the cause and the date of the decease.

Results: three hundred fifty six patients were interviewed; $66(18.53 \%)$ stated at least one adverse event and $21(31.80 \%)$ events occurred in over 60 years-old patients. Eighty seven adverse effects were recorded; $8(9.20 \%)$ comprised infections such as hepatitis $B$, hepatitis $C$, foliculosis, mononucleosis and warts, one $(1.10 \%)$ event corresponded to cancer and $78(89.70 \%)$ to other type of events. Their relationship with the administration of the drug was considered as conditional in 64 events $(73.60 \%)$ and as unlikely in 21 (24.10\%) events comprising infections and most of the fatal events.

Conclusions: the adverse events observed in the patients, some years after the end of the treatment with the transfer factor, were not considered to be related to the administration of this drug; however, they must be under surveillance since the information obtained from this study should be compared with the information collected by a higher number of observations and of controlled observational studies.

Key words: transfer factor, adverse reactions, adverse events, drug surveillance and drug epidemiology.

\section{NTRODUCCI ÓN}

La farmacovigilancia es una de las estrategias de la Farmacoepidemiología, se ocupa de identificar y valorar los efectos del uso de los medicamentos en la población, sobre todo las reacciones adversas que provocan. Se desarrolla después que estos se registran para su comercialización y durante todo el tiempo que permanezcan en el mercado, pues existen diferencias entre los resultados de los ensayos clínicos previos 
al registro y los que se observan después en la práctica médica, básicamente porque un mayor número de personas se exponen al fármaco, sin las condiciones especiales que se dan en estos ensayos y durante un mayor tiempo. ${ }^{1}$

El factor de transferencia (FT) es un hemoderivado que se emplea en el tratamiento de la inmunodeficiencia celular, consiste en un extracto dializable de leucocitos que transfiere inmunidad de un donante inmune a otro con déficit inmunológico; se produce en el Centro de Ingeniería Genética y Biotecnología (CIGB) en La Habana, Cuba y se comercializa con el nombre de Hebertrans ${ }^{\circledR} .^{2,3}$

Son escasos los estudios publicados que describen o evalúan las reacciones adversas del FT en las condiciones de la práctica médica habitual, así como cualquier evento que pueda aparecer tras su empleo; ${ }^{4,5}$ menos información hay sobre aquellos que requieren tiempo para expresarse, como el cáncer.

Los productores en el $\mathrm{ClGB}$, refieren que el medicamento solo provoca un ligero eritema en el sitio de la inyección en $2 \%$ de los casos y el sistema cubano de farmacovigilancia registra fiebre, frialdad, palidez y otras raras como petequias, vómitos, erupción cutánea, fatiga, cianosis, adenopatías, eritema, celulitis y prurito. ${ }^{6}$ Una investigación señala que cerca de la cuarta parte de las terapias biológicas han estado sujetas al menos a una acción reguladora relacionada con la seguridad durante la década siguiente a su aprobación. Se informa riesgo de transmisión de infecciones, tumores, pérdida de la visión y papiledema, leucoencefalopatía multifocal progresiva, linfoma, daño hepático y otros eventos para diferentes medicamentos biológicos, años después de su entrada al mercado. ${ }^{7-9}$ En la producción de Hebertrans ${ }^{\circledR}$ se realiza una cuidadosa selección de los donantes y análisis de la materia prima para detectar marcadores virales, se evalúa el producto en momentos críticos del proceso de producción y se valida la capacidad del proceso para inactivar o remover los posibles contaminantes virales presentes en las materias primas, ${ }^{2}$ pero en la práctica clínica no se conoce el resultado de estos procesos.

La escasa información sobre la seguridad del FT justifica la necesidad de vigilar su uso, para contribuir a establecer su perfil de reacciones adversas y así su relación beneficios/riesgos.

El presente informe extiende la vigilancia realizada al FT contenida en diferentes publicaciones, que abordan, cómo se prescribe el fármaco, ${ }^{10}$ la evolución clínica de los pacientes tratados, ${ }^{11}$ los eventos adversos que se presentaron durante su administración y los observados al año siguiente, al término del tratamiento. ${ }^{4,5}$

En esta publicación, los objetivos propuestos son, identificar eventos adversos presentados años después de finalizado el tratamiento con FT y determinar si existe algún grado de asociación entre el fármaco y los eventos observados.

\section{MÉTODOS}

Se realizó un estudio de tipo observacional, retrospectivo, de farmacovigilancia activa. ${ }^{12,13}$

El universo estuvo constituido por 413 pacientes tratados con este fármaco por sus médicos de asistencia entre los años 2001 y 2004 en 11 hospitales de La Habana: cinco hospitales clinicoquirúrgicos, tres hospitales pediátricos y tres institutos del tercer nivel de atención sanitaria. De los 413 pacientes, 57 quedaron fuera del estudio 
por la imposibilidad de entrevistarlos, por lo que la muestra quedó conformada por 356 pacientes.

El periodo de observación abarcó de 6 a 8 años posteriores al tratamiento, en función de los diversos esquemas de tratamiento que se utilizaron. ${ }^{10}$

Se estudió la presencia de hepatitis $\mathrm{C}$ o B, VIH, cáncer y otros eventos, en estos últimos se incluyó cualquier nuevo diagnóstico o motivo surgido en el periodo de estudio, que llevara a la remisión del paciente a un especialista, o que fuera hospitalizado, o cualquier otra experiencia no deseada, pero lo suficientemente importante como para ser aceptada. ${ }^{12,13} \mathrm{Si}$ el evento fue la muerte del paciente, se entrevistó al familiar para registrar la causa y fecha de la muerte.

Para analizar el grado de asociación con la administración de FT, se tuvo en cuenta el tiempo transcurrido entre el tratamiento y la aparición del evento, las enfermedades asociadas, los medicamentos consumidos de forma crónica, si hubo reexposición al FT $\mathrm{u}$ otros hemoderivados en el periodo de estudio y la edad del paciente.

Los eventos detectados se analizaron por un comité de expertos integrado por tres autores y dos especialistas de la Unidad Nacional Coordinadora de Farmacovigilancia, para valorar el grado de asociación con el FT, se tuvieron en cuenta las categorías empleadas en The Uppsala Monitoring Centre: definitiva, probable, posible, condicional, improbable. ${ }^{12,13}$

Los pacientes fueron localizados y entrevistados en su hogar por los autores, se empleó un cuestionario estructurado confeccionado al efecto. Si en la tercera visita no se lograba obtener la información, quedaba fuera del estudio. La entrevista se realizó con el consentimiento del paciente o un familiar en caso de los niños.

Los datos se analizaron mediante distribución de frecuencias y porcentajes.

\section{RESULTADOS}

Se entrevistaron 356 pacientes. Se registró al menos un evento adverso en 66 pacientes, $18,53 \%$ del total de entrevistados.

En la tabla 1 se observa que del total de pacientes con eventos, la mayoría se detectó en aquellos con edades iguales o mayores de 60 años, 21 (31,8\%) y entre 45 y 59 años, $19(28,8 \%)$.

La tabla 2 muestra que se registraron 87 eventos adversos, de ellos 8 (9,2 \%) fueron infecciones, $1(1,1 \%)$ cáncer y $78(89,7 \%)$ otros eventos.

En el periodo de estudio fallecieron 12 pacientes, las causas fueron infarto agudo del miocardio en 4 casos, cáncer de pulmón en 3 y coma alcohólico, infección respiratoria y tromboembolismo pulmonar en un caso, respectivamente; en 2 pacientes no se pudo saber la causa. La mayoría de estos pacientes tenían más de 60 años y diversas enfermedades crónicas asociadas.

En los niños los eventos registrados fueron epilepsia en 3 casos y asma bronquial, cefaleas frecuentes, gastritis, hipertensión arterial, insuficiencia renal, miopía, trombopatía, verrugas vulgares y mononucleosis en un caso, respectivamente. 
Tabla 1. Pacientes según eventos adversos registrados años después del tratamiento con factor de transferencia y grupos de edad

\begin{tabular}{|c|c|c|c|c|c|c|}
\hline \multirow{2}{*}{ Edad (años) } & \multicolumn{2}{|c|}{ Sin eventos } & \multicolumn{2}{|c|}{ Con eventos } & \multicolumn{2}{|c|}{ Total } \\
\hline & No. & $\%$ & No. & $\%$ & No. & $\%$ \\
\hline 0 a 14 & 60 & 20,8 & 12 & 18,2 & 72 & 20,3 \\
\hline 15 a 29 & 74 & 25,5 & 7 & 10,6 & 81 & 22,7 \\
\hline 30 a 44 & 38 & 13,1 & 7 & 10,6 & 45 & 12,6 \\
\hline 45 a 59 & 59 & 20,3 & 19 & 28,8 & 78 & 21,9 \\
\hline 60 y más & 59 & 20,3 & 21 & 31,8 & 80 & 22,5 \\
\hline Total & 290 & 100 & 66 & 100 & 356 & 100 \\
\hline
\end{tabular}

Tabla 2. Eventos adversos registrados años después del tratamiento con factor de transferencia según tipo de evento

\begin{tabular}{|c|c|c|c|}
\hline $\begin{array}{l}\text { Tipo } \\
\text { de evento }\end{array}$ & Evento adverso (cantidad de pacientes) & No. & $\%$ \\
\hline Infecciones & $\begin{array}{l}\text { Hepatitis C (3), hepatitis B (2), verrugas vulgares } \\
\text { (1), mononucleosis (1), foliculosis (1) }\end{array}$ & 8 & 9,2 \\
\hline Cáncer & Cáncer de mama (1) & 1 & 1,1 \\
\hline $\begin{array}{l}\text { Otros } \\
\text { eventos }\end{array}$ & $\begin{array}{l}\text { Muerte (12), hipertensión arterial (8), diabetes mellitus } \\
\text { (8), asma bronquial (4), glaucoma (4), epilepsia ( } 3 \text {, } \\
\text { gastritis (2), insuficiencia renal (2), enfermedad celiaca } \\
\text { (2), artritis reumatoide (2), cefaleas frecuentes (1), } \\
\text { miopía (1), trombopatía (1), anemia (1), condromalacia } \\
\text { (1), escoliosis (1), litiasis renal (1), obesidad (1), } \\
\text { síndrome de mala absorción (1), alergia a alimentos } \\
\text { (1), pie equino en hijo de madre expuesta (1), cataratas } \\
\text { (1), litiasis vesicular (1), dermatitis seborreica (1), } \\
\text { enfisema (1), fibromialgia (1), migraña (1), úlcera } \\
\text { gástrica (1), hiperesplenismo (1), desprendimiento de } \\
\text { retina (1), hipoacusia (1), enfermedad cerebrovascular } \\
\text { (1), bronquiectasia (1), hernia hiatal (1), insomnio (1), } \\
\text { cardiopatía isquémica (1), colitis ulcerativa (1), neumpatía } \\
\text { (1), esclerodermia (1), pólipos y divertículos intestinales } \\
\text { (1), psoriasis (1) }\end{array}$ & 78 & 89,7 \\
\hline \multicolumn{2}{|l|}{ Total } & 87 & 100 \\
\hline
\end{tabular}

Al analizar la asociación entre los eventos y la administración de FT, ninguno se consideró como una reacción adversa definitiva, probable o posible, porque la información obtenida no fue suficiente para establecer estas categorías.

En la tabla 3 se aprecia que 64 eventos (73,6 \%), tenían una causalidad condicional, principalmente porque la secuencia temporal entre el inicio del evento y el evento en sí era razonable, pero se requerían más datos para su análisis. Por ejemplo, dos pacientes fallecieron por cáncer de pulmón 3 y 5 años después de la exposición al FT, pero la información disponible fue insuficiente para considerar otra clasificación, uno de ellos era un hombre de 78 años, que padecía úlcera gástrica y fue tratado con FT por presentar infecciones oftalmológicas a repetición, el otro caso fue una mujer de 59 años con antecedentes de asma bronquial, enfermedad pulmonar obstructiva crónica, insuficiencia renal y alergias a medicamentos, que fue tratada con FT por

http://scielo.sld.cu 
sepsis respiratorias a repetición. También se evaluó como condicional el evento cáncer de mama, diagnosticado 6 años después del tratamiento con FT, en una paciente de 52 años con antecedentes de hipertensión arterial, diabetes mellitus, alergias y queratocono, que recibió el medicamento por presentar queratoconjuntivitis alérgica.

Tabla 3. Eventos adversos registrados años después del tratamiento con factor de transferencia según asociación de causalidad

\begin{tabular}{|c|c|c|c|}
\hline Causalidad & Evento adverso (cantidad de pacientes) & No. & $\%$ \\
\hline Condicional & $\begin{array}{l}\text { Hipertensión arterial (8), diabetes mellitus (8), asma } \\
\text { bronquial (4), glaucoma (4), epilepsia ( } 3 \text {, muerte (2), } \\
\text { gastritis (2), enfermedad celiaca (2), artritis reumatoide (2), } \\
\text { cáncer de mama (1), insuficiencia renal (1), cefaleas } \\
\text { frecuentes (1), trombopatía (1), miopía (1), anemia (1), } \\
\text { litiasis renal (1), obesidad (1), síndrome de mala absorción } \\
\text { (1), alergia a alimentos (1), pie equino en hijo de madre } \\
\text { expuesta (1), cataratas (1), litiasis vesicular (1), dermatitis } \\
\text { seborreica (1), enfisema (1), fibromialgia (1), migraña (1), } \\
\text { ulcera gástrica (1), hipoacusia (1), enfermedad cerebro- } \\
\text { vascular (1), bronquiectasia (1), hernia hiatal (1), insomnio } \\
\text { (1), cardiopatía isquémica (1), psoriasis (1), colitis ulcerativa } \\
\text { (1), neuropatía (1), esclerodermia (1), pólipos y divertículos } \\
\text { intestinales (1) }\end{array}$ & 64 & 73,6 \\
\hline Improbable & $\begin{array}{l}\text { Muerte (8), hepatitis B (2), hepatitis C (3), condromalacia (1), } \\
\text { escoliosis (1), foliculosis (1), mononucleosis (1), verrugas } \\
\text { vulgares (1), desprendimiento de retina (1), hiperesple- } \\
\text { nismo (1), insuficiencia renal (1) }\end{array}$ & 21 & 24,1 \\
\hline No evaluable & Muerte (2) & 2 & 2,3 \\
\hline \multicolumn{2}{|l|}{ Total } & 87 & 100 \\
\hline
\end{tabular}

Los eventos clasificados como improbables, 21 (24,1 \%), no tuvieron una secuencia temporal razonable, además podían ser explicados de forma más plausible por las enfermedades concurrentes o por otros fármacos consumidos por el paciente. Por ejemplo, las infecciones se presentaron entre 4 y 6 años después de la exposición al FT, la mayoría en pacientes adultos con antecedentes de presentar infecciones a repetición antes del uso de FT y que se habían expuesto a otros hemoderivados en el periodo analizado.

La mononucleosis se presentó en un niño de 13 años que ya en una ocasión anterior a su exposición al FT había padecido esta enfermedad, además estuvo en contacto con otros fármacos por padecer de esofagitis distal crónica y el evento se manifestó a los 2 años de recibir el FT, por lo que resultó difícil establecer una asociación con el fármaco. Se consideró también improbable que la muerte de 8 pacientes estuviera asociada con el FT, pues la causa se correspondía más con la enfermedad de base o con sus cuadros característicos. Por ejemplo cuatro pacientes que fallecieron por infarto del miocardio, mujeres de 54, 71, 77 y 88 años, tenían antecedentes de hipertensión arterial y otros factores de riesgo cardiovascular y las muertes se produjeron 5 a 7 años después del tratamiento; otro caso fue un hombre de 69 años con antecedentes de hipertensión arterial, enfermedad pulmonar obstructiva crónica y alergia al yodo, que falleció por tromboembolismo pulmonar 6 años después del tratamiento con FT.

Otras muertes consideradas improbables fueron la de un hombre de 54 años por cáncer de pulmón, quien padecía de esta enfermedad antes del tratamiento, otro 
hombre de 53 años por coma alcohólico y otro de 69 años que falleció por bronconeumonía, 3 años después del tratamiento y con antecedentes de infecciones respiratorias frecuentes. Dos eventos fatales se consideraron no evaluables porque no se pudo conocer la causa de muerte ni otra información sobre el periodo de estudio. Uno de estos pacientes era un niño de 15 años que padecía de asma bronquial, epilepsia, encefalopatía, infecciones a repetición y alergias, recibió el tratamiento por infecciones respiratorias a repetición. El otro caso correspondió a un hombre de 59 años, que recibió tratamiento por presentar infecciones respiratorias a repetición, sin otros antecedentes patológicos.

\section{DISCUSI ÓN}

Años después de haber recibido tratamiento con $\mathrm{FT}$, los eventos adversos que se registraron en los pacientes tratados no se consideraron asociados con su administración, resultados que sugieren su inocuidad.

Sin embargo, estos eventos deben ser vigilados, pues la información obtenida en este estudio debe ser contrastada con un mayor número de observaciones, para confirmar o descartar cualquiera de ellos, porque la fuerza de la asociación de causalidad aumenta si el evento se ha informado por otros. ${ }^{12,13}$ También deben ser verificados en estudios observacionales controlados, que lamentablemente no se han publicado.

Un sesgo de memoria por parte de los pacientes pudo estar presente en esta investigación, pues se preguntaba retrospectivamente sobre los eventos presentados y los medicamentos consumidos en ese periodo. El hecho de preguntar por enfermedades graves como infecciones de transmisión hemática y cáncer pudo compensarlo, pues difícilmente los pacientes o familiares olvidarían dichos eventos, aunque sí sus detalles.

Similar a lo que refiere la literatura, ${ }^{12}$ los eventos detectados fueron más frecuentes en el adulto mayor y en los niños, también se registró un número importante de eventos, por lo que estas poblaciones deben ser las más vigiladas por los profesionales sanitarios.

La probabilidad de que un medicamento biológico reciba una advertencia de seguridad es de $14 \%$ después de los 3 años en el mercado y aumenta a $29 \%$ a los 10 años, aunque casi el $50 \%$ de ellas están relacionadas con trastornos generales, con la vía de administración parenteral y con las infecciones. ${ }^{7}$

La etapa poscomercialización de Hebertrans ${ }^{\circledR}$ tiene más de 20 años y hasta el momento no se conoce de advertencias graves sobre su seguridad, se han reconocido escasas reacciones adversas, ${ }^{4-6}$ y la mayoría de los eventos registrados en este estudio, valorados como condicionales, se presentaron entre 2 y 3 años después del tratamiento. Estos resultados justifican la presencia de FT en el cuadro básico de medicamentos de Cuba, pero los prescriptores deben tener en cuenta que su seguridad no está establecida y por tanto este fármaco debe utilizarse solo en las indicaciones aprobadas para su uso.

A pesar de que los eventos registrados no se asociaron con la administración de factor de transferencia, la vigilancia de su uso debe continuar mientras este medicamento permanezca en el mercado farmacéutico. 


\section{REFERENCI AS BI BLI OGRÁFICAS}

1. Furones J A, Pérez J. Necesidad de la Farmacoepidemiología. Farmacoepidemiología. Uso racional de medicamentos. La Habana: Editorial Academia; 2010. p. 1-14.

2. Fernández CB. Factor de transferencia, nuevas actividades biológicas. Avances Médicos Cuba. 1999; 7:30-2.

3. Simposio Internacional sobre Interferón y Citocinas [Internet]. La Habana: Centro de Ingeniería Genética y Biotecnología; 2001 dic [citado 21 diciembre 2007].

Disponible en: http://elfosscientiae.cigb.edu.cu/PDFs/BA/2001/18/Especial /BA00180E013-024.pdf

4. Cruz MA, Rodríguez BN, Furones JA, Alonso L, Calvo DM, Rego JJ. Efectos adversos asociados al tratamiento con factor de transferencia. Ciudad de La Habana, 2004. Rev Cubana Farm [Internet]. 2006 [citado 23 abril 2010]; 40(1). Disponible en: http://bvs. sld.cu/revistas/far/vol40_1_06/far04106. htm

5. Cruz MA, Rodríguez BN, Furones J A, Alfonso I, Rodríguez D. Eventos adversos observados después del tratamiento con factor de transferencia. Rev Cubana Salud Pública [Internet]. 2012 [citado 9 enero 2012];38(1). Disponible en:

http://bvs.sld.cu/revistas/spu/vol38_1_12/spu03112.htm

6. Colectivo de autores. Factor de transferencia [Internet]. La Habana: Formulario Nacional de Medicamentos; 2011 [citado 3 marzo 2012]. Disponible en: http://fnmedicamentos. sld. cu/index.php?P=FullRecord\&ID=380

7. Giezen TJ, Mantel-Teeuwisse AK, Straus SMJ M, Schellekens $H$, Leufkens HGM, Egberts ACG. Safety-related regulatory actions for biological approved in the United States and the European Union. JAMA [Internet]. 2008 [cited 2010 Jul 11];300(16): 1887-96. Available from: http://jama. ama-assn.org/cgi/content/full/300 $\angle 16 / 1887$

8. Bongartz T, Sutton AJ, Sweeting MJ, Buchan I, Matteson EL, Montori V. Anti-TNF antibody therapy in rheumatoid arthritis and the risk of serious infections and malignancies: systematic review and meta-analysis of rare harmful effects in randomized controlled trials. JAMA [Internet]. 2006 [cited 2010 J ul 23];295(19):2275-85. Available from: http://jama. ama-assn. org/cgi/content/full/295 $\angle 19 / 2275$

9. Papa A, Mocci G, Bonizzi M, Felice C, Andrisani G, Para G, et al. Biological therapy for inflammatory bowel disease: controversies and future options. Expert Rev Clin Pharmacol. 2009; 2 (4):391-403.

10. Cruz MA, Rodríguez BN, Furones J A, Martín AD, Guerra LM, Páez AT. Patrones de prescripción de factor de transferencia en 11 hospitales de Ciudad de La Habana, año 2002. Rev Cubana Salud Pública [Internet]. 2005 [citado 23 Abr 2010]; 31(4).

Disponible en: http://bvs.sld.cu/revistas/spu/vol31_4_05/spu04405.htm

11. Cruz MA, Rodríguez BN, Furones JA, Pérez E, Morris AC, Huete S. Evolución clínica de pacientes tratados con factor de transferencia. Rev Cubana Hematología [Internet]. 2004 [citado $23 \mathrm{Abr} 2010$ ];20(3). Disponible en:

http://bvs.sld.cu/revistas/hih/vol20_3_04/hih06304.htm

http://scielo.sld.cu 
12. Furones JA. Reacciones adversas. Farmacovigilancia e interacciones medicamentosas. En: Vergel Rivera G, Tasé Martínez MJ, Groning Roque E, editores. Farmacología en el proceso de atención de enfermería. La Habana: Editorial Ciencias Médicas; 2009. p. 52-88.

13. Vigilancia de la seguridad de los medicamentos. Guía para la instalación y puesta en funcionamiento de un centro de farmacovigilancia [Internet]. WHO: Uppsala Monitoring Centre; 2001 [citado 24 May 2002]. Disponible en:

http://whoumc.org/graphics/24751.pdf

Recibido: 30 de abril de 2012.

Aprobado: 31 de julio de 2012.

María Aida Cruz Barrios. Facultad de Medicina "Miguel Enríquez", Universidad de Ciencias Médicas de La Habana. Calle Ramón Pintó No. 202, Luyanó, municipio "10 de Octubre". La Habana, Cuba.

Correo electrónico: maria.cruz@infomed.sld.cu 